\title{
Baixo peso e dependência funcional em idosos institucionalizados de Uberlândia (MG), Brasil
}

\author{
Low weight and functional disability in institutionalized elderly \\ interns in Uberlândia in the State of Minas Gerais, Brazil
}

Kamilla Tavares de Sousa ${ }^{1}$

Laura Anália Silva de Mesquita ${ }^{1}$

Leandro Alves Pereira ${ }^{2}$

Catarina Machado Azeredo ${ }^{1}$

${ }^{1}$ Faculdade de Medicina, Universidade Federal de Uberlândia. Av. Pará 1720/ Bloco 2U/sala 23, Campus Umuarama. 38.405-320 Uberlândia MG Brasil. kamillatavares100@

hotmail.com

${ }^{2}$ Faculdade de Matemática, Universidade Federal de

Uberlândia.

\begin{abstract}
This article seeks to identify the association between nutritional status and functional dependence in institutionalized elderly interns in Uberlândia, Minas Gerais. It involved a cross-sectional study with 233 elderly interns in which sociodemographic variables, nutritional status and degree of dependency were assessed. Multivariate analysis was performed using Poisson regression with robust variance to assess the association between nutritional status and functional dependence of these senior citizens. Low weight by the Body Mass Index was associated with functional dependence after controlling for socioeconomic and demographic variables. The elderly with low weight had a prevalence ratio of dependence of 1.2 times the prevalence rate of dependence of well nourished elderly interns. As reported in the literature, age was also associated with dependence with a prevalence ratio of 1.19 for the longest living. Low weight is an important factor associated with functional disability. Thus, the need for evaluation, monitoring and recovery of nutritional status in the institutionalized elderly is stressed, as in addition to reducing morbidity and mortality from several diseases and improve quality of life, it can also prevent and delay functional dependency.

Key words Activities of daily life, Functional capacity, Nutritional status, Health of the elderly, Nursing homes
\end{abstract}

Resumo O objetivo deste artigo é identificar a associação entre estado nutricional e dependência funcional dos idosos institucionalizados de Uberlândia (MG). Estudo transversal, com 233 idosos. Foram avaliadas variáveis sociodemográficas, estado nutricional e o grau de dependência dos idosos. Realizou-se análise multivariada pela Regressão de Poisson com variância robusta, para verificar a associação entre o estado nutricional e dependência funcional. O baixo peso pelo Índice de Massa Corporal se associou à dependência funcional após controle para variáveis socioeconômicas e demográficas, os idosos com baixo peso tinham uma razão de prevalência de dependência de 1,20 vezes àquela dos eutróficos. Como bem reportado pela literatura, a idade também se associou à dependência, com razão de prevalência de 1,19, para os longevos. O baixo peso constituiu importante fator associado à incapacidade funcional. Dessa forma, ressalta-se a necessidade de avaliação, monitoramento e recuperação do estado nutricional de idosos institucionalizados, que além de reduzir morbimortalidade por diversas doenças e melhorar a qualidade de vida, ainda pode prevenir e retardar a dependência funcional.

Palavras-chave Atividades cotidianas, Capacidade funcional, Estado nutricional, Saúde do idoso, Instituições de longa permanência para idosos 


\section{Introdução}

O envelhecimento populacional é um fenômeno cada vez mais presente em nossa sociedade ${ }^{1}$, o que constitui um grande desafio, pois acarreta em aumento de demanda para os serviços de saúde, de previdência social e de recursos humanos para cuidar da população idosa² ${ }^{2}$.

Esse processo de envelhecimento pode levar a incertezas quanto às condições de cuidado desses indivíduos, fazendo com que muitas famílias optem por dividir a responsabilidade do cuidado com as chamadas Instituições de Longa Permanência para Idosos (ILPI) ${ }^{3}$.

Além disso, sabe-se que apesar de natural, o envelhecimento é um processo que submete o organismo a um conjunto de alterações anatômicas e funcionais, que repercutem no estado de saúde e nutrição, e com grande frequência leva a redução da capacidade funcional e a alterações nos processos metabólicos do organismo ${ }^{4}$, os quais poderão contribuir para o aumento da morbimortalidade desses indivíduos 5 .

Dentre os problemas comumente desencadeados pelo avançar da idade destaca-se a incapacidade funcional, entendida como um conjunto de alterações que acarretam maior dependência de cuidadores ${ }^{6}$.

A incapacidade funcional tem como principais fatores de risco a presença de problemas neurológicos, alterações nutricionais e sedentarismo ${ }^{6}$, e traz importantes prejuízos à saúde e à qualidade de vida dos idosos, reduzindo a capacidade de autocuidado e algumas vezes a mobilidade, ocasionando baixa autoestima, depressão e, consequentemente, menor vontade de viver ${ }^{7}$. Segundo Sposito et al. ${ }^{8}$, com o aumento da expectativa de vida e as modificações físicas, psicológicas e sociais acarretadas pelo envelhecimento, é de fundamental importância que se propicie ao idoso uma boa qualidade de vida, sendo a independência funcional um dos fatores limitantes para essa questão.

A desnutrição é outro problema comum na terceira idade, sendo considerado o distúrbio mais importante nesta fase da vida. A desnutrição pode contribuir para o aumento da mortalidade, aumento da susceptibilidade às infecções e a redução da qualidade de vida dos idosos. Além do mais, tem como consequência a diminuição da força muscular, da capacidade de ação e da aptidão cardiorrespiratória, contribuindo ainda mais para a incapacidade funcional ${ }^{6,9}$.

A desnutrição tem causa multifatorial e está associada a problemas de saúde comumente observados na terceira idade, como as Doenças Crônicas não Transmissíveis (DCNT), deficiências físicas e cognitivas, depressão, perda de apetite, problemas de mastigação, dificuldades de deglutição e a própria falta de independência para se alimentar ${ }^{10}$. Sendo assim, no que concerne à relação entre desnutrição e a incapacidade funcional, observa-se que a mesma pode ser bidirecional, com reforço mútuo entre as condições.

Para a obtenção de menores taxas de desnutrição e consequentemente maior independência nas Atividades de Vida Diária (AVD) na população idosa é preciso que os indivíduos mantenham um bom estado nutricional alcançado a partir de uma alimentação adequada ${ }^{11}$.

A presença de incapacidade funcional é mais relevante em idosos que residem em ILPI, quando comparados idosos não institucionalizados ${ }^{12}$, e há escassez de estudos baseados em população institucionalizada. Assim, objetivou-se identificar a associação entre o estado nutricional e o nível de dependência para a realização das atividades de vida diária dos idosos institucionalizados da cidade de Uberlândia (MG).

A compreensão sobre o grau de associação entre esses fatores pode favorecer a elaboração de propostas que visem à melhoria da saúde, do estado nutricional e da qualidade de vida destes sujeitos.

\section{Metodologia}

Trata-se de um estudo transversal, realizado nas ILPI de Uberlândia (MG), sobre a situação nutricional e a capacidade de realização das Atividades Básicas de Vida Diária dos idosos institucionalizados.

Todas as 15 ILPI existentes no município de Uberlândia, reconhecidas pelo Conselho Municipal de Saúde do Idoso, aceitaram participar deste estudo. O total de idosos que viviam nas ILPI no momento do estudo era de 289, destes 56 não participaram da pesquisa, sendo os principais motivos: recusa ou ausência nas três visitas da equipe em diferentes momentos. Dessa forma foram avaliados os demais 233 idosos que tiveram o termo de consentimento livre e esclarecido assinado por eles ou pelo responsável da ILPI. O período de coleta de dados foi de outubro de 2010 a outubro de 2011.

Para o presente estudo não se utilizou amostra, uma vez que todos os idosos institucionalizados foram convidados, de modo a atender solicitação das respectivas coordenadorias. Dessa forma, não foram realizadas as inferências estatísticas (testes de hipóteses pelo p-valor e construção de 
intervalos de confiança), pois consideramos o erro amostral nulo, já que se trata de um censo dos idosos institucionalizados de Uberlândia.

Diante disso, considera-se que os resultados obtidos são representativos da população idosa institucionalizada de Uberlândia, pois o número de indivíduos avaliados foi muito próximo do total de residentes nas ILPI, de forma que as estimativas amostrais seriam muito parecidas caso tivesse sido coletado dados de todos.

Para a coleta de dados foram utilizados três instrumentos: 1) um questionário elaborado pelos pesquisadores contendo informações demográficas, de saúde geral e dados de avaliação nutricional objetiva: idade, sexo, estado civil, escolaridade, religião, tipo de ILPI, tempo de internação na ILPI, presença de problemas de saúde (foram considerados quaisquer doenças relatadas pelos idosos, cuidadores ou registrados no prontuário do indivíduo) e IMC calculado pela fórmula peso $(\mathrm{kg}) /$ estatura ${ }^{2}(\mathrm{~m})$; 2) A Mini Avaliação Nutricional (MAN) para avaliação nutricional subjetiva - MAN; 3) o protocolo de Índice de Katz para avaliação das AVD.

Para a avaliação do estado nutricional foi aferido o peso - com o uso de balança digital portátil, e estatura - com uso de estadiômetro compacto para fixação em paredes. Os idosos eram pesados com vestimentas leves e o estadiômetro foi instalado em paredes que não apresentavam rodapés, para evitar erros de aferição.

Para os idosos acamados ou os que apresentaram algum problema postural, foram aferidas as dobras cutâneas triciptal e subescapular com o adipômetro Lange ${ }^{\oplus} \mathrm{e}$ altura do joelho com uso de estadiômetro de madeira, para estimativa de peso e estatura a partir da equação de Chumlea et al. ${ }^{13}$. O IMC foi calculado por meio da divisão do peso pela altura elevada ao quadrado.

A classificação do IMC dos idosos foi realizada de acordo com os pontos de corte propostos pela Organização Pan-Americana de Saúde (OPAS) ${ }^{14}$, da seguinte forma: baixo peso (IMC $\leq 23 \mathrm{~kg} / \mathrm{m}^{2}$ ), peso normal ou eutrofia $\left(23<\mathrm{IMC}<28 \mathrm{~kg} / \mathrm{m}^{2}\right)$, pré-obesidade ou sobrepeso $(28 \leq \mathrm{IMC}<30 \mathrm{~kg} /$ $\mathrm{m}^{2}$ ) e obesidade (IMC $\geq 30 \mathrm{~kg} / \mathrm{m}^{2}$ ).

Além da antropometria utilizou-se a MAN, método subjetivo para a detecção precoce de risco nutricional especificamente para a população geriátrica. A MAN consiste em um instrumento de 18 itens, que abrange quatro dimensões (antropometria, avaliação dietética, avaliação global e autoavaliação). Os itens da primeira etapa (etapa de triagem) se destinaram a detecção dos idosos "com risco de desnutrição", e com estes indivíduos foi realizado o restante do questionário (etapa de avaliação global). Os escores obtidos classificaram os idosos como eutróficos, com risco de desnutrição ou desnutridos ${ }^{15}$.

Para avaliar a capacidade funcional dos idosos foi utilizado o protocolo Index de ADL de Katz ${ }^{16}$, o qual avaliou seis parâmetros (banhar-se, vestirse, ir ao banheiro, transferência, continência e alimentação). Os idosos recebiam pontuação " 1 " em cada parâmetro caso fossem independentes ou pontuação " 0 " caso dependessem de auxílio para realizar a tarefa. Após a avaliação dos parâmetros foi feito o somatório dos pontos e os idosos foram classificados como independentes (pontuação $\geq$ 5), com dependência moderada (pontuação entre 3 e 4) ou muito dependentes (pontuação $\leq 2$ ).

Os idosos conscientes responderam as perguntas dos questionários, enquanto aqueles com problemas neurológicos ou incapazes de se comunicar tiveram as questões respondidas pelos cuidadores. A equipe responsável pela coleta de dados passou por capacitações sobre o correto manuseio e aplicação dos instrumentos e dos equipamentos utilizados.

Os dados foram analisados no Software $\mathrm{R}$ for Windows (pacote "Rcmdr"). A variável de desfecho do presente estudo foi a "dependência funcional", criada a partir do somatório de idosos classificados com dependência moderada com os muito dependentes. As variáveis de exposição foram divididas entre demográficas (idade e sexo - coletadas nos prontuários dos idosos), relativas à institucionalização (tempo que residia à ILPI, tipo de ILPI - privada ou filantrópica) e aspectos relacionados à saúde e nutrição (presença de doenças, se já sofreu queda ou fratura e estado nutricional pelo IMC). A variável idade foi categorizada para ser incluída no modelo ( $<80$ anos ou 80 anos ou mais), considerando que nos idosos longevos a dependência ocorre com maior frequência e que dividir os idosos em três ou mais categorias de idade não traria benefícios e reduziria o poder da análise devido ao número total de idosos. Foram coletados dados referentes à escolaridade, entretanto, aproximadamente metade dos idosos não tinham registros sobre essa informação e não souberam relata-la. Portanto, a variável não foi incluída no modelo de regressão múltipla.

Inicialmente fez-se uma análise descritiva dos dados, para todas as variáveis acima citadas. Posteriormente realizou-se análise bivariada com o intuito de verificar associação entre as variáveis de exposição ao desfecho, e a partir do coeficiente de correlação de Spearman (Rho) definiu-se a inclusão das variáveis no modelo multivariado 
sempre que Rho $\geq 0,05$ ( $\geq 5 \%$ ) ou que a variável tivesse importância biológica (como sexo e idade). A análise múltipla foi realizada por Regressão Poisson com variância robusta para a obtenção da razão de prevalências, considerando que o desfecho apresentou elevada prevalência (> 20\%) e que, portanto, o odds ratio não seria um índice adequado para descrever a influência dos fatores de exposição.

A pesquisa foi conduzida dentro dos preceitos éticos preconizados pela Declaração de Helsinque e pela Resolução CNS 466-2012 $2^{17}$, além das normas do Comitê de Ética em Pesquisa da Universidade Federal de Uberlândia.

\section{Resultados}

A população estudada apresentou uma média de idade de 79,5 anos ( $\mathrm{DP}=9,98)$, sendo a maioria do sexo feminino $(62,7 \%, \mathrm{n}=146)$, viúvos $(44,2 \%$, $\mathrm{n}=103)$ e residentes em instituições filantrópicas $(56,2 \%, \mathrm{n}=131)$.

Em relação à saúde dos idosos institucionalizados, $87,1 \%$ deles apresentavam algum problema de saúde, sendo os mais frequentes: hipertensão arterial sistêmica $(39,9 \%, \mathrm{n}=93)$, problemas mentais/demências $(32,2 \%, \mathrm{n}=75)$ e problemas cardíacos $(11,2 \%, \mathrm{n}=26)$. Neste contexto, 94,0\% $(\mathrm{n}=219)$ faziam uso de algum medicamento e $37,3 \%(n=87)$ já haviam sofrido alguma queda e/ou fratura (Tabela 1).

A Tabela 2 apresenta a caracterização dos participantes do estudo, incluindo o estado nutricional dos idosos segundo o IMC e a MAN, com destaque para o baixo peso pelo IMC $(58,8 \%, \mathrm{n}=$ $137)$, para o risco de desnutrição $(41,6 \%, \mathrm{n}=97)$ e desnutrição $(26,2 \%, \mathrm{n}=61)$ pela MAN.

Quanto ao grau de dependência, observou-se que apenas 33,0\% ( $\mathrm{n}=77)$ dos idosos institucionalizados eram independentes em suas AVD, e os outros $67,0 \%(n=156)$ apresentaram algum grau de dependência. Dentre estes, 10,7\% ( $\mathrm{n}=$ 25) tinham dependência moderada e 56,2\% (n = 131) eram muito dependentes.

A análise bivariada (Tabela 3 ) permitiu constatar que, a princípio, as variáveis que mais se associaram à dependência funcional, de forma isolada, foram: idade (maior dependência entre os mais velhos); estado nutricional pelo IMC (maior dependência entre idosos com baixo peso); estado nutricional pela MAN (maior dependência entre idosos com risco de desnutrição e desnutridos).

Os idosos desnutridos ou que tinham risco para desnutrição, segundo a classificação da MAN,
Tabela 1. Caracterização socioeconômica, demográfica e aspectos de saúde dos idosos institucionalizados de Uberlândia, MG, 2010.

\begin{tabular}{lr}
\hline \multicolumn{1}{c}{ Variáveis } & Participantes n (\%) \\
\hline Faixa etária & $109(46,8)$ \\
$<80$ anos & $124(53,2)$ \\
80 anos ou mais & \\
Sexo & $87(37,3)$ \\
Masculino & $146(62,6)$ \\
Feminino & \\
Escolaridade & $29(12,4)$ \\
Analfabeto & $64(27,5)$ \\
Até 8 anos de estudo & $17(7,3)$ \\
Mais de 8 anos de estudo & $123(52,8)$ \\
Não sabe/Não respondeu & $24(10-60)$ \\
Tempo de internação (meses) & \\
Tipo de ILPI & $131(56,2)$ \\
Filantrópica & $102(43,8)$ \\
Particular & \\
Problema de saúde & $30(12,9)$ \\
Não & $203(87,1)$ \\
Sim & $130(55,8)$ \\
Queda ou fratura & $87(37,4)$ \\
Não & $16(6,8)$ \\
Sim & \\
Não sabe/Não respondeu & \\
\hline
\end{tabular}

"mediana (intervalo interquartil).

Tabela 2. Caracterização do estado nutricional e dependência funcional dos idosos institucionalizados de Uberlândia, MG, 2010.

\begin{tabular}{lc}
\hline \multicolumn{1}{c}{ Variáveis } & Participantes n (\%) \\
\hline Estado nutricional IMC & $74(31,8)$ \\
Eutrófico & $137(58,8)$ \\
Baixo peso & $10(4,3)$ \\
Sobrepeso & $12(5,1)$ \\
Obesidade & \\
Estado nutricional MAN & $75(32,2)$ \\
Eutrófico & $97(41,6)$ \\
Sob risco de desnutrição & $61(26,2)$ \\
Desnutridos & \\
Dependência funcional & $77(33,0)$ \\
Independentes & $25(10,7)$ \\
Dependência moderada & $131(56,2)$ \\
Muito dependentes & \\
\hline
\end{tabular}

"mediana (intervalo interquartil).

apresentavam média de idade de 81,0 ( $\mathrm{DP}=10,4)$ anos, enquanto os que não apresentavam esse risco tinham uma média de idade menor, de 76,5 $(\mathrm{DP}=8,3)$ anos. $\mathrm{O}$ mesmo pode ser observado na questão do grau de dependência, pois os idosos mais dependentes tinham média de idade de 80,6 
$(\mathrm{DP}=10,0)$ anos e os que eram independentes em suas AVD tinham média de idade de 77,5 (DP $=9,7$ ) anos.

Dessa forma, os idosos sem risco de desnutrição eram em média 4,5 anos mais novos em relação aos que apresentaram algum desvio nutricional, e os idosos independentes eram em média três anos mais novos que os dependentes. Tais resultados reforçaram a necessidade de inclusão da variável faixa etária no modelo da análise múltipla, obtendo-se uma RP =1,19 (Tabela 4). A variável sexo também foi incluída no modelo para ajuste.

Os idosos classificados com baixo peso pelo IMC obtiveram uma maior proporção de risco para a dependência funcional $(R P=1,20)$. Porém, o excesso de peso não conferiu maior risco para essa característica (Tabela 4).

Os dados sobre a MAN não foram incluídos no modelo multivariado, em função de haver questões de mobilidade e funcionalidade que são semelhantes aos itens avaliados no Protocolo de Katz.

\section{Discussão}

A transição demográfica, advinda do envelhecimento populacional, está diretamente relacionada com a transição epidemiológica, em que aumenta o número de DCNT e diminuem os casos de doenças infecciosas e parasitárias. Segundo Chaimowicz ${ }^{18}$ as doenças mais frequentes nos idosos atuais são os acidentes vasculares cerebrais, fraturas após quedas, insuficiência cardíacas e/ou pulmonares, diabetes mellitus e Alzheimer.

Nesse sentido, em um estudo com a população idosa do Sudeste brasileiro ${ }^{19}$, foi visto que cerca de $86 \%$ destes indivíduos apresentava algum tipo de doença, principalmente as DCNT, semelhante ao encontrado em nosso estudo.

Além das DCNT, a queda é uma causa externa responsável por elevada mortalidade que pode causar sequelas irreversíveis, contribuir para a redução da capacidade funcional e consequentemente para a dependência de cuidadores. No pre-

Tabela 3. Análise bivariada de características demográficas, de saúde e estado nutricional com dependência funcional, em idosos institucionalizados de Uberlândia, MG, 2010.

\begin{tabular}{|c|c|c|c|}
\hline Variáveis & $\begin{array}{c}\text { Participantes n (\%) } \\
\text { sem dependência }\end{array}$ & $\begin{array}{l}\text { Participantes com } \\
\text { dependência } \mathbf{n}(\%)\end{array}$ & Rho $^{*}$ \\
\hline \multicolumn{4}{|l|}{ Sexo } \\
\hline Masculino & $29(33,3)$ & $58(66,7)$ & \multirow[t]{2}{*}{0,005} \\
\hline Feminino & $48(32,9)$ & $98(67,1)$ & \\
\hline \multicolumn{4}{|l|}{ Faixa etária } \\
\hline$<80$ anos & $44(40,4)$ & $65(59,6)$ & \multirow[t]{2}{*}{0,146} \\
\hline 80 anos ou mais & $33(26,6)$ & $91(73,4)$ & \\
\hline Tempo de internação (meses) & $24(9-72)$ & $24(11-57)$ & -0.144 \\
\hline \multicolumn{4}{|l|}{ Tipo de ILPI } \\
\hline Filantrópica & $46(35,1)$ & $85(64,9)$ & \multirow[t]{2}{*}{0,050} \\
\hline Particular & $31(30,4)$ & $71(69,6)$ & \\
\hline \multicolumn{4}{|l|}{ Problema de Saúde } \\
\hline Não & $10(33,3)$ & $20(66,7)$ & \multirow[t]{2}{*}{0,002} \\
\hline Sim & $67(33,0)$ & $136(67,0)$ & \\
\hline \multicolumn{4}{|l|}{ Estado nutricional IMC } \\
\hline \multirow[t]{2}{*}{ Eutrófico (E) } & \multirow[t]{2}{*}{$30(40,5)$} & \multirow[t]{2}{*}{$44(59,5)$} & $0,150(\mathrm{E}-\mathrm{B})$ \\
\hline & & & $0,130(\mathrm{E}-\mathrm{S})$ \\
\hline \multirow[t]{2}{*}{ Baixo peso (B) } & \multirow[t]{2}{*}{$35(25,5)$} & \multirow[t]{2}{*}{$102(74,5)$} & $-0,070(\mathrm{E}-0)$ \\
\hline & & & $-0,190(\mathrm{~B}-\mathrm{S})$ \\
\hline \multirow[t]{2}{*}{ Sobrepeso (S) } & \multirow[t]{2}{*}{$6(60,0)$} & \multirow[t]{2}{*}{$4(40,0)$} & $-0,150(B-0)$ \\
\hline & & & $0,100(\mathrm{~S}-0)$ \\
\hline Obesidade $(\mathrm{O})$ & $6(50,0)$ & $5(50,0)$ & \\
\hline \multicolumn{4}{|l|}{ Estado Nutricional MAN } \\
\hline Eutrófico & $40(53,3)$ & $35(46,7)$ & \\
\hline Sob risco de desnutrição & $27(27,8)$ & $70(72,2)$ & \\
\hline Desnutrição & $10(16,4)$ & $51(83,6)$ & 0,310 \\
\hline \multicolumn{4}{|l|}{ Queda ou fratura } \\
\hline Não & $42(32,3)$ & $88(67,7)$ & \\
\hline Sim & $32(36,8)$ & $55(63,2)$ & $-0,040$ \\
\hline
\end{tabular}

* teste de correlação de Spearman. 
Tabela 4. Análise multivariada da associação entre dependência funcional e fatores de risco, em idosos institucionalizados de Uberlândia, MG, 2010.

\begin{tabular}{|c|c|c|}
\hline Variável & Coeficiente & $\begin{array}{l}\text { Razão de } \\
\text { Prevalência } \\
\quad(\text { RP) }\end{array}$ \\
\hline Intercepto & -0.570 & \\
\hline \multicolumn{3}{|l|}{ Sexo } \\
\hline Masculino & & 1 \\
\hline Feminino & $-0,040$ & 0,96 \\
\hline \multicolumn{3}{|l|}{ Faixa etária } \\
\hline$<80$ anos & 0,180 & 1 \\
\hline 80 anos ou mais & & 1,19 \\
\hline Tempo de Internação (meses) & $-0,001$ & 1,0 \\
\hline \multicolumn{3}{|l|}{ Tipo de ILPI } \\
\hline Filantrópica & 0,030 & 1 \\
\hline Particular & & 1,03 \\
\hline \multicolumn{3}{|l|}{ Estado Nutricional IMC } \\
\hline Eutrófico & & 1 \\
\hline Baixo Peso & 0,180 & 1,20 \\
\hline Sobrepeso & $-0,400$ & 0,67 \\
\hline Obesidade & $-0,230$ & 0,80 \\
\hline
\end{tabular}

"Regressão Poisson múltipla com variância robusta.

sente estudo, não se observou associação entre as quedas e a dependência funcional, provavelmente porque grande parte dos idosos já havia sofrido quedas e a gravidade deste evento não foi avaliada. A elevada frequência de quedas nos sujeitos estudados vai ao encontro do estudo de Teixeira et al. ${ }^{20}$, que observou que cerca de 30 a $40 \%$ dos idosos institucionalizados em Belo Horizonte já sofreram queda ao solo pelo menos uma vez na vida. Dados relativamente mais baixos foram vistos por Pereira et al..$^{21}$, os quais observaram $10,7 \%$ de quedas entre os idosos estudados, vale ressaltar que o estudo deles não foi realizado com sujeitos institucionalizados.

No que tange à dependência funcional de idosos, dados da literatura mostram achados bastante semelhantes aos que encontramos. As pesquisas de Araújo e Ceolim ${ }^{22}$ e de Barbosa ${ }^{23}$, com idosos institucionalizados, encontraram que $37 \%$ e $38 \%$ dos idosos, respectivamente, eram independentes em suas AVD, assim como no presente trabalho que identificou a proporção de $33 \%$. Desta forma, estes dados contribuem para manter a imagem de que a institucionalização ainda está, muitas vezes, associada à dependência física e cognitiva dos idosos.

Outro fator importante para a dependência funcional é a idade, visto que com o passar dos anos tende a aumentar. No estudo de Araújo e Ceolim ${ }^{22}$, os idosos independentes também apresentavam média de idade menor que 80 anos, assim como os aqueles avaliados nas ILPI de Uberlândia. Considerando que o avanço da idade influencia no declínio da capacidade funcional dos idosos, devem ser envidados esforços no sentido de prevenir a dependência física, e retardá-la o máximo possível, para que possam viver por mais tempo com uma boa qualidade de vida ${ }^{24 .}$

Além da incapacidade física, o baixo peso é outro problema que afeta em grande proporção a população idosa. Busnello ${ }^{25}$ destaca que os idosos brasileiros têm mais chances de desenvolver deficiências nutricionais, por se tratar de um país em desenvolvimento e em que muitas questões de assistência à saúde ainda precisam ser melhoradas. O mesmo autor corroborou com nossos achados ao avaliar o estado nutricional de idosos residentes em ILPI, observando prevalências de baixo peso, segundo IMC, entre 25 e $60 \%$.

A alta prevalência de desnutrição em idosos institucionalizados, avaliados por meio da MAN, também foi mostrada por Sperotto e Spinelli ${ }^{26}$, em que $35 \%$ estavam desnutridos e $65 \%$ com risco de desnutrição. Valores relativamente mais baixos, que os encontrados em nosso estudo, foram achados por Sousa e Lovisi ${ }^{27}$, que encontraram uma prevalência de $12,5 \%$ de idosos com desnutrição e 32,8\% com risco de desnutrição.

Para esta população específica, o excesso de peso apresentou baixa prevalência, dado comumente encontrado em idosos institucionalizados, assim como mostrado por Menezes e Marucci ${ }^{28}$ e por Rauen ${ }^{29}$. Além de pouco prevalente, o excesso de peso não interferiu na dependência dos idosos, porém, não foram encontrados trabalhos na literatura que permitissem a discussão destes achados. Em um estudo com idosas que apresentavam sobrepeso, mas que não residiam em ILPI, encontrou-se um índice de aptidão física abaixo do normal. Contudo, a metodologia adotada por esses pesquisadores foi diferente da que adotamos, sendo utilizados instrumentos que avaliaram a agilidade física, coordenação e resistência, variáveis que podem ser prejudicadas pelo excesso de peso ${ }^{30}$.

Não obstante, o baixo peso representou importante fator de risco para a dependência dos idosos, independente dos outros fatores analisados. Isso pode ser explicado pelo fato de que para realizar qualquer atividade que exija esforço físico, como as AVD, é necessário que a massa corporal esteja preservada. Outros estudos também observaram que indivíduos com depleção de massa (desnutridos ou com baixo peso), na maioria das vezes não 
estão aptos para executar diversas atividades do seu cotidiano, determinando uma maior dependência dos idosos desnutridos comparados aos com excesso de peso $^{31-33}$.

Ressalta-se que a depleção de massa magra também pode acontecer em indivíduos obesos ( $\mathrm{fe}$ nômeno conhecido como obesidade sarcopênica problema decorrente do avançar da idade e da perda de neurônios motores, acarretando na restrição do funcionamento do sistema neuromuscular), em que ocorre o declínio de força e consequentemente leva a incapacidade funcional. Essas mudanças são decorrentes de uma grande vulnerabilidade fisiológica, perda involuntária de massa muscular e aumento de gordura corporal ${ }^{34,35}$.

Dessa forma, a ausência de dados sobre sarcopenia foi uma limitação do estudo, visto que a associação entre esta e a dependência é bastante comum. Entretanto, optou-se por avaliar o estado nutricional por meio do IMC e MAN por se tratar de instrumentos de mais fácil acesso aos profissionais de saúde e profissionais das ILPI, permitindo um acompanhamento contínuo do estado nutricional e do grau de dependência, e da associação entre eles nos idosos. Outra limitação é o delineamento transversal, o que impede inferir sobre a direção da associação entre as variáveis, entretanto, a literatura aponta o baixo peso como responsável por perdas importantes na capacidade funcional de idosos, levando a diminuição de grande parte da capacidade motora, funcional e intelectual, o que acarreta em maior dependência de cuidadores ${ }^{5,36,37}$. Este fato, não descarta a hipótese de que a dependência interfira na desnutrição, por comprometer a capacidade do indivíduo de se alimentar, que se constitui uma das AVD.

Outra questão a ser destacada é que não foram obtidos dados dos indivíduos não participantes do estudo (perdas) e, portanto, não foi possível compará-los aos idosos participantes. Dessa forma, embora as perdas estejam em um limite aceitável (19\%), não é possível afastar totalmente a hipótese de que difiram dos idosos incluídos em relação a alguma característica que implique na não representatividade.

\section{Conclusões}

O perfil dos idosos institucionalizados caracteriza-se por uma população predominantemente feminina, viúva, analfabeta ou com poucos anos de estudo e residente em instituições filantrópicas. Torna-se evidente a maior fragilidade desse grupo populacional, por se tratar de idosos longevos, com várias comorbidades e dependentes de medicamentos, o que muitas vezes constitui motivo para a institucionalização.

Embora a progressão da idade influencie naturalmente a capacidade funcional e o estado nutricional dos indivíduos, os idosos apresentaram sério comprometimento desses indicadores de saúde.

Mesmo sendo uma avaliação pontual dos idosos institucionalizados de Uberlândia (MG) a literatura permite inferir que o estado nutricional influencia a capacidade funcional, e o presente estudo demonstra claramente a necessidade de prevenção e reabilitação do baixo peso, bem como de ações que visem prevenir e retardar a dependência física pelo maior tempo possível.

O presente estudo pode ser utilizado como importante subsidio para cuidadores e demais profissionais da saúde que trabalham em ILPI, na elaboração de planos de cuidados adequados e atualizados, respeitando sempre a capacidade funcional de cada idoso como forma de assegurarlhe qualidade de vida.

\section{Colaboradores}

KT Sousa, LAS Mesquita e CM Azeredo elaboraram o projeto de pesquisa. KT Sousa e LAS Mesquita realizaram a coleta de dados. LA Pereira e CM Azeredo realizaram as análises estatísticas dos dados. Todos os autores elaboraram a versão final do artigo científico. 


\section{Referências}

1. Brasil. Ministério da Saúde (MS). Envelhecimento e Saúde da Pessoa Idosa. Cad Atenção Básica 2006; 19:192.

2. Papaléo Netto M, Ponte JR. Gerontologia: A velhice e o envelhecimento em visão globalizada. $2^{\text {a }}$ ed. São Paulo: Atheneu; 2002. p. 3-12.

3. Camarano AA, Kanso S. As instituições de longa permanência para idosos no Brasil. Rev Bras Estud Popul 2010; 27(1):233-235.

4. Campos MTS, Monteiro JBR, Ornelas APRC. Fatores que afetam o consumo alimentar e a nutrição de idosos. Rev Nutr 2000; 13(3):157-165.

5. Acunã K, Cruz T. Avaliação do estado nutricional de adultos e idosos e situação nutricional da população brasileira. Arq Bras Endocinol Metab 2004; 48(3):345-361.

6. Strobl R, Muller M, Emeny R, Peters A, Grill E. Distribution and determinants of functioning and disabiliti in aged adults - results from the German KORA-Age study. BMC Public Health 2013; 13:137.

7. Caldas CP. Envelhecimento com dependência: responsabilidades e demandas da família. Cad Saude Publica 2003; 19(3):773-781.

8. Sposito G, D’Elboux MJ, Neri AL, Guariento ME. A satisfação com a vida e a funcionalidade em idosos atendidos em um ambulatório de geriatria. Cien Saude Colet 2013; 18(12):3475-3482.

9. Ghisla MK, Cossi S, Timpini A, Baroni F, Facchi E, Marengoni A. Predictors of successful rehabilitation in geriatric patients: subgroup analysis of patients with cognitive impairment. Aging Clin Exp Res 2007; 19(5):417-423.

10. Mamhidir A-G, Kihlgren M, Soerlie V. Malnutrition in elder care: qualitative analysis of ethical perceptions of politicians and civil servants. BMC Medical Ethics 2010; 11:11.

11. Guigoz Y, Vellas B, Garry PJ. Assessing the nutritional status of the elderly: The Mini Nutritional Assessments part of the geriatric evaluation. Nutr Rev 1996; 54(1 $\mathrm{Pt}): 59-65$.

12. Vellas BJ, Alberede JL, Garry PJ. Diseases and aging: Patterns of morbidity with age; relationship between aging and age-associated diseases. Am J Clin Nutr 1992; 55(Supl. 6):1225-1230.

13. Chumlea WC, Guo S, Roche AF, Steivaugh ML. Estimating stature from knee height for person 60 to 90 years of age. J Am Med Assoc 1985; 33(2):116-120.

14. Organização Pan-Americana de Saúde (OPAS). XXXVI Reunión del Comitê Asesor de Investigaciones en Salud - Encuestra Multicêntrica - Salud Beinestar y Envejecimeiento (SABE) en América Latina e el CaribeInforme preliminar. [acessado 2012 dez 5] Disponível em URL: http://www.imsersomayores.csic.es/documentos/ documentos/paho-salud-01.pdf

15. Sampaio LR. Avaliação nutricional e envelhecimento. Rev Nutr 2004; 17(4):507-514.

16. The Hartford Institute For Geriatric Nursing. Katz Index of Independence in Activities of Daily Living (ADL). [Internet]. 1998 [acessado 2012 jul 6]. Disponível em URL: http://www.hartfordign.org/.

17. Brasil. Ministério da Saúde (MS). Conselho Nacional de Saúde. Resolução no 466, de 12 de dezembro de 2012. Diário Oficial da União 2013, 13 jun.

18. Chaimowicz F. A saúde dos idosos brasileiros às vésperas do século XXI: problemas, projeções e alternativas. Rev Saude Publica 1997; 31(2):184-200.
19. Ramos LR, Rosa TEC, Oliveira ZM, Medina MCG, Santos FRG. Perfil do idoso em área metropolitana na região sudeste do Brasil: resultados de inquérito domiciliar. Rev Saude Publica 1993; 27(2):87-94.

20. Teixeira DC, Oliveira IL, Dias RC. Perfil demográfico, clínico e funcional de idosos institucionalizados com história de quedas. Fisioter Mov 2006; 9(2):101-108.

21. Pereira GN, Morsh P, Lopes DGC, Trevisan MD, Ribeiro A, Navarro JHN, Bós DSG, Viana MSS, Bós AJG. Fatores socioambientais associados à ocorrência de quedas em idosos. Cien Saude Colet 2013; 18(12):3507-3514.

22. Araújo MOPH, Ceolim MF. Avaliação do grau de independência de idosos residentes em instituições de longa permanência. Rev Esc Enferm USP 2007; 41(3):378-385.

23. Barbosa MLJ. A situação dos idosos no Vale do Paraíba: análise nas cidades de Taubaté e Guaratinguetá [tese]. São Paulo: Universidade de São Paulo; 1990.

24. Paschoal S, Má P. Epidemiologia do Envelhecimento. In: Papalèo Netto M, organizador. Gerontologia: a velhice e o envelhecimento em visão globalizada. São Paulo: Atheneu; 1996. p. 26-43.

25. Busnello FM. Aspectos nutricionais no processo do envelhecimento. São Paulo: Atheneu; 2007.

26. Sperotto FM, Spinelli RB. Avaliação nutricional em idoso independentes de uma instituição de longa permanênia no município de Erechim - RS. Perspectiva, Erechim 2010; 34(125):105-116.

27. Sousa RG, Lovisi GM. Avaliação de déficits cognitivos em moradores com mais de 65 anos de um albergue público. Rev Psiquiatr Clín 2007; 34(5):205-209.

28. Menezes TN, Marucci MFN. Antropometria de idosos residentes em instituições geriátricas, Fortaleza, CE. Rev Saude Publica 2005; 39(2):169-175.

29. Rauen MS. Avaliação do estado nutricional de idosos institucionalizados. Rev Nutr PUCCAMP 2008; 21(3):303-310.

30. Rech CR, Cruz JLS, Araújo EDS, Kalinowski FG, Dellagrama RA. Associação entre aptidão funcional e excesso de peso em mulheres idosas. Motricidade 2010; 6(2):47-53.

31. Benn SJ, McCartney N, McKelvie RS. Circulatory responses to weight lifting, walking, and stair climbing in older males. J Am Geriatr Soc 1996; 44(2):121-125.

32. Brill PA, Macera CA, Davis DR, Blair SN, Gordon N. Muscular strength and physical function. Med Sci Sports Exerc 2000; 32(2):412-416.

33. Andre MB, Dumavibhat N, Ngatu NR, Eitoku M, Hirota R, Suganuma N. Mini Nutritional Assessment and functional capacity in community-dwelling elderly in Rural Luozi, Democratic Republic of Congo. Geriatr Gerontol Int 2013; 13(1):35-42.

34. Funes JAA, Mayo EJG. Beneficios de la prática del ejercicio em los ancianos. Gac Méd Mex 2004; 140(4):431-436.

35. Maior AS. Relação sarcopenia e treinamento de força. Rev Fisioter: UNICid, São Paulo 2004; 3(2):125-139.

36. Incalzi RA, Pegano F, deBruno E, Landi F, Cipriani L, Carbonin P. Malnutrition in the acute care hospital: a very common problem. Ann Ital Med Int 1995; 10(4):222-226.

37. Cigolle CT, Langa KM, Kabeto MU, Tian Z, Blaum CS. Geriatric conditions and disability: the Health and Retirement Study. Ann Intern Med 2007; 147(3):156-164.

Artigo apresentado em 30/10/2013

Aprovado em 31/03/2014

Versão final apresentada em 07/04/2014 